\title{
The hierarchical structure of seashells optimized to resist mechanical threats
}

\author{
M. Yourdkhani, D. Pasini \& F. Barthelat \\ Department of Mechanical Engineering, McGill University, Canada
}

\begin{abstract}
The vast majority of mollusks grow a hard shell for protection. Typical seashells are composed of two distinct layers, with an outer layer made of calcite, which is a hard but brittle material, and an inner layer made of a tough and ductile material called nacre. Nacre is a biocomposite material that consists of more than $95 \%$ of tablet-shaped aragonite, $\mathrm{CaCO}_{3}$, and a soft organic material as the matrix. Although the brittle ceramic aragonite constitutes a high volume fraction of nacre, its mechanical properties are found to be surprisingly higher than those of its constituents. Calcite and nacre, two materials with distinct structures and properties, are believed to be arranged in an optimal fashion to defeat attacks from predators. This paper aims at capturing the design rules of a gastropod seashell by using multiscale modeling and optimization techniques. A two-layer finite element model of the seashell was developed to include shell geometry at the macroscale, whereas nacre material properties were modeled at the microscale. A representative volume element of the microstructure of nacre was used to formulate a closed-form expression of the elastic modulus of nacre, and a multiaxial failure criterion as a function of the key dimensions of the microstructure. Using the seashell model, the maximum load that the shell can carry at its apex was obtained and different failure modes were introduced. The results from optimization suggested that the natural seashell is optimally designed for resisting penetrations. Furthermore, experiments were performed on an actual shell of abalone to validate the results obtained from simulations and gain insight into the way that the shell fails under sharp penetration. Optimization and experimental results revealed that the shell shows its best performance when two modes of failure coincide within the structure.

Keywords: seashell, multiscale modeling, representative volume element, failure criterion, nacre, multiscale optimization.
\end{abstract}




\section{Introduction}

Seashells are hard biological structures that are believed to be optimally designed for protection against mechanical threats, and they are now considered as a potential source of inspiration for biomimetics [1]. A seashell is essentially a hard ceramic layer used for protection of the soft tissues of the mollusk animal. Many gastropod and bivalve shells are composed of two distinct materials: an outer layer made of calcite and the inner iridescent layer, which is composed of nacre. Calcite is a prismatic ceramic material made of calcium carbonate $\left(\mathrm{CaCO}_{3}\right)$, a hard but brittle material. On the other hand, nacre is a tough and ductile material, which shows relatively large plastic deformation prior to failure [2-4]. The combination of a hard layer on the outside with a tougher, more ductile layer on the inside is considered to provide an ideal protective structure $[2,4]$. When a seashell is exposed to a concentrated load, for example a predator's bite, the hard ceramic layer can prevent penetration, while the inside layer can absorb excesses of mechanical energy. In the case of overloading, the brittle calcite layer may fail, due to cracks propagating toward the mollusk's soft tissue. Experiments have demonstrated that the tough nacreous layer could delay and eventually arrest those cracks, thereby delaying the overall shell failure.

To date, extensive research has been performed on the structure and properties of nacre and calcite but no study has investigated how these two materials operate together in the actual shell. In particular, while there is evidence that nacre is optimized for toughness and energy absorption, little is known about how the shell structure makes the most of its material constituents, i.e., calcite and nacre. The hierarchical structure of the seashell as observed in a natural specimen is the result of a long multiscale optimization process, from which we expect to gain insight useful to the design of similar biomimetic protective structures. In this work, a multiscale modeling is performed on the structure of seashell to understand how the hierarchical structure plays a key role in resisting attacks from natural predators.

\section{Seashell modeling}

The hierarchical structure of red abalone is represented by its macroscale geometry, while its constituent materials are governed at microscale. In order to investigate the multiscale structural and material characteristics of the seashell, a model of the structure of red abalone shell was created in ANSYS (ANSYS, Inc., Canonsburg, PA). The real geometry of a shell can be quite complex; in this work, a simplified geometry was used to retain the following features: the shell is composed of two layers and it is curved. It is assumed that (i) the shell has a uniform thickness; (ii) its shape can be approximated with a spherical cap and (iii) the periphery of the shell is in full contact with the support. Based on these assumptions, an axisymmetric two-layer model of the seashell was developed in ANSYS (fig. 1). In this model, the outer and inner layers represent calcite and nacre, with corresponding material properties, respectively. The plane-42 


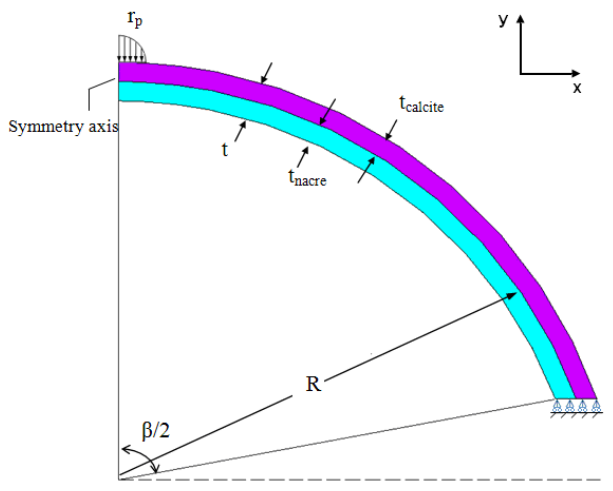

Figure 1: $\quad$ Two-layer model of a seashell.

element was used in ANSYS to mesh the model. The mesh was refined at the region under the area in contact with the external loading to yield the accurate result. In fig. 1, $R$ is the radius of curvature of the shell (taken at the nacre/calcite interface), $t$ is the thickness of the shell and $\beta$ is the opening angle of the spherical cap. In order to model a sharp contact load, e.g., from a predator's tooth, a spherical distribution of pressure consistent with contact stresses was imposed on a small region at the apex of the shell [5]. The corresponding applied pressure can be written as:

$$
P(r)=\frac{3}{2} P_{m}\left(1-\left(\frac{r}{r_{p}}\right)^{2}\right)^{1 / 2}
$$

where $r_{p}$ is the radius of contact load and $r$ is the distance from an arbitrary point of the distributed load area to the symmetry axis of the shell; $P_{m}$ is the average pressure within the contact region.

The outer layer of the shell was modeled with material properties of calcite with a Young's modulus of $100 \mathrm{GPa}$ and Poisson's ratio of 0.3 Caspi et al. [6]. The principal stress in calcite was used to predict its brittle failure. It was assumed that failure occurred when the largest principal stress in any point of the calcite layer exceeds its maximum strength, which is $100 \mathrm{MPa}$ [6]. The inner layer of the shell is composed of nacre, whose mechanical properties depend on its microstructure. In order to correlate the mechanical properties of nacre with its microstructure, a micromechanical model of nacre was developed, which will be explained in detail in the following section.

\subsection{Microscale modeling of nacre}

Nacre has an organized and well-designed brick and mortar microstructure [4] (fig. 2a). The flat polygonal "bricks" or "tablets" are separated and bonded by 
a)

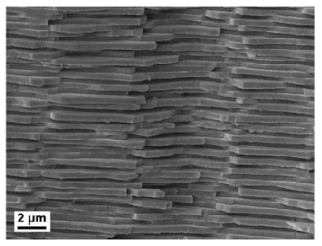

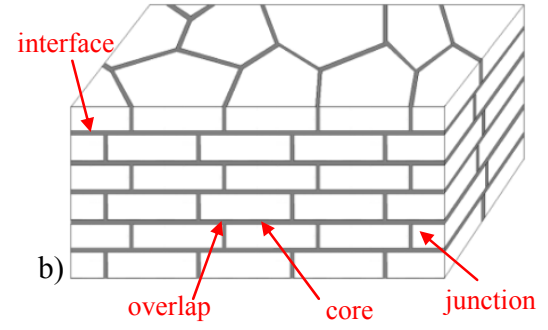

Overlap

c)

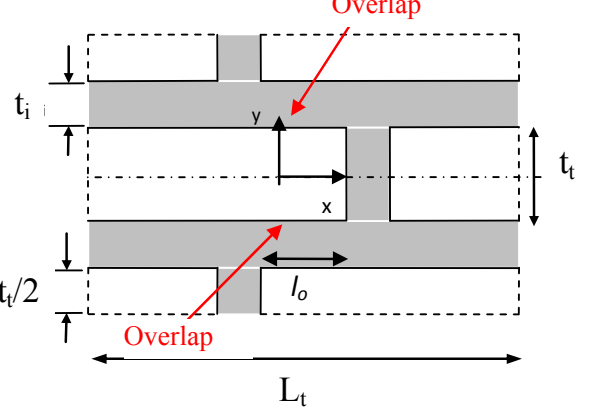

Figure 2: Microstructure of nacre: a) scanning electron micrograph of a fractured surface of nacre (Barthelat et al. [2]); b) schematic of tablet arrangement in nacre; c) schematic of representative volume element.

soft organic materials (less than $5 \%$ in volume). Hereafter, the inter-lamella distance is called the interface and the inter-tablet gap is referred to as the junction (fig. 2b) [2]. It is also known that the size and the aspect ratio of the tablets in nacre vary from one seashell to another [7]. For example, in nacre from red abalone, the average tablet diameter and thickness are around $8 \mu \mathrm{m}$ and $0.4 \mu \mathrm{m}$, respectively [2], whereas the nacre of Pinctada (oyster) shell tablets are around $4 \mu \mathrm{m}$ long and $0.5 \mu \mathrm{m}$ thick [8]. The thickness of the organic interface in sheet and columnar nacre was found to be about 20 to $30 \mathrm{~nm}$, being small compared to that of a single tablet $[2,9]$. In columnar nacre, the tablets are arranged in columns and they are arranged in two distinct regions, the "overlap" and "core", defined in Barthelat et al. [2]. The "overlap" is the region where two adjacent tablets in two neighboring layers overlap with each other (fig. 2b). The remaining part of each tablet, which is not covered in the overlapping region, is referred to as the "core" [2]. The overlap region in columnar nacre covers around $1 / 3$ of the area of a tablet. Another type of nacre is sheet nacre, where the arrangement of the tablets is more random.

The mechanical properties of nacre strongly depend on its microstructure. In order to capture that dependence, an appropriate micromechanics model is required to express the properties of nacre in terms of its microstructural geometry and material properties. A representative volume element (RVE) is generally used to model the mechanical behavior of nacre $[8,10]$ (fig. 2c). In the 
RVE, $t_{i}, t_{t}, L_{t}$ and $l_{o}$, represent the thickness of the interface, the thickness of the tablet, tablet length and overlap length, respectively. In order to simplify the analysis while retaining the salient mechanisms in the material, the following assumptions are typically considered:

- The mechanical response of this model is representative of the behavior of the whole material. The model is periodic along both directions $\mathrm{x}$ and $\mathrm{y}$, in terms of geometry, displacements and stress.

- The tablets have uniform thickness. In the plane of tablets, they have a squared cross section.

- The tablets carry only axial stresses, while the interfaces only transfer shear stresses, so the applied stress is carried by a series of shear and tensile elements.

- Axial and shear stresses are assumed to be uniform across the thickness of the tablets and interface.

- Plane stress distribution is considered in the calculations, as the tablets have finite dimensions and the soft organic interface has the effect of relaxing the constraints applied by the $x-y$ and $x-z$ tablet surfaces, which thus behave as free surfaces. As a result, the effect of Poisson's ratio is neglected.

\subsubsection{In-plane elastic modulus}

An analytical model of the in-plane elastic modulus of nacre was first proposed by Kotha et al. [8]. The solution was obtained for fully overlapped nacre by neglecting the effect of junctions between two adjacent tablets of one layer. Since the overlap region transfers axial stress between the tablets, its length plays an important role on the total stress transfer as well as on the elastic modulus. Here, the Kotha's expression is revisited to include arbitrary overlap lengths and junction effects. In order to make the mechanical properties of nacre independent of its microscale dimensions, the microstructural dimensions were normalized with respect to the length of the tablet.

$$
\bar{t}_{t}=\frac{t_{t}}{L_{t}}, \bar{t}_{i}=\frac{t_{i}}{L_{t}}, \bar{t}_{j}=\frac{t_{j}}{L_{t}}, \bar{l}_{o}=\frac{l_{o}}{L_{t}}
$$

The resulting in-plane elastic modulus of nacre is found to be:

$$
E_{\text {In-plane }}=\frac{\bar{t}_{t}}{\overline{\bar{t}_{t}}+\overline{t_{i}}} \frac{1}{\left(\frac{1}{E_{t}}+\frac{\bar{t}_{j}}{E_{i}} \frac{N}{1+N}\right)}
$$

where $N$ is

$$
N=\left(\frac{\alpha \bar{t}_{t} \bar{t}_{i}}{2 \bar{t}_{j}} \frac{E_{i}}{G_{i}}\right)\left[\frac{1+\cosh \left(\alpha \bar{l}_{o}\right)}{\sinh \left(\alpha \bar{l}_{o}\right)}+\frac{1+\cosh \left(\alpha\left(1-\bar{l}_{o}\right)\right)}{\sinh \left(\alpha\left(1-\bar{l}_{o}\right)\right)}\right]
$$

and 


$$
\alpha=\sqrt{\frac{4 G_{i}}{\overline{t_{t} \bar{t}_{i}} E_{t}}}
$$

Out-of-plane elastic and shear moduli were formulated using the Reuss composite model (Barthelat et al. [11]) and in-plane shear modulus was formulated by assuming isotropic property in the plane of the tablet.

$$
G_{\text {In-plane }}=\frac{E_{\text {In-plane }}}{2(1+v)}
$$

where $v$ is the corresponding Poisson's ratio with a value of 0.2 [12].

\subsubsection{Failure criterion}

The choice of an appropriate failure criterion is required to suitably predict the failure of nacre under various loading conditions. None of the main failure criteria used for composite materials could match the experimental data available for nacre. Instead, a micromechanics-based multiaxial failure criterion was derived for nacre. In this work, we focus on the failure in tension along the tablets as observed through experiments. Failure of the organic interface was considered to be the key reason for the failure of nacre in shear and tension across the tablets. We assume the material fails when any point of the RVE fails.

The basic idea to derive the failure criterion is to model the yielded interface with a series of parallel springs that can be stretched along and across the tablets to the extent defined by the finite strength of the organic material. As a result, the failure criterion was obtained as

$$
\sigma_{y y}{ }^{2}+\left(\frac{\sigma_{x x}\left(\bar{t}_{t}+\bar{t}_{i}\right)-\sigma_{i}{ }^{y} \bar{t}_{t}}{\bar{l}_{o}}+\left|\tau_{x y}\right|\right)^{2}=\left(\sigma_{i}{ }^{y}\right)^{2}
$$

where $\sigma_{i}^{y}$ is the junction yield stress.

\subsection{Failure criterion for the shell}

For a given load, the finite element model returns the corresponding stresses in the two layers. A "safety factor" $(\lambda)$ was calculated for both layers to detect the critical point of the shell. For the calcite layer, $\lambda$ is defined as ratio of principal stress to the calcite ultimate strength whereas for nacre, $\lambda$ is calculated using the previously derived failure criterion, eqn (8). For a given load, the stresses and consequently safety factor for all points of the structure are calculated. The point with the highest $\lambda$ is prone to failure ahead of other points. Based on this approach, failure can occur at different locations on the shell depending on the geometrical parameters of the shell Lawn et al. [13]. The thickness of the shell and the amount of calcite in the structure were found to be the key parameters in the locating the failure. As a result, the following failures may happen in the shell. 

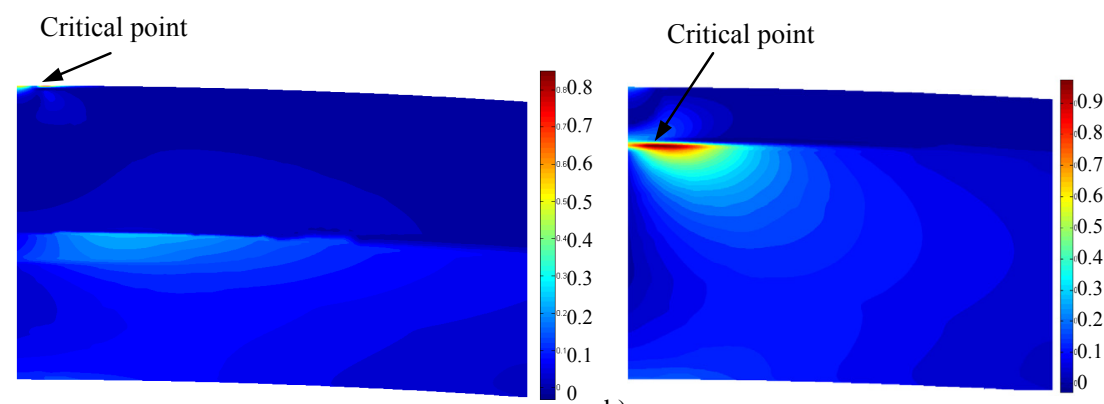

a)

b)

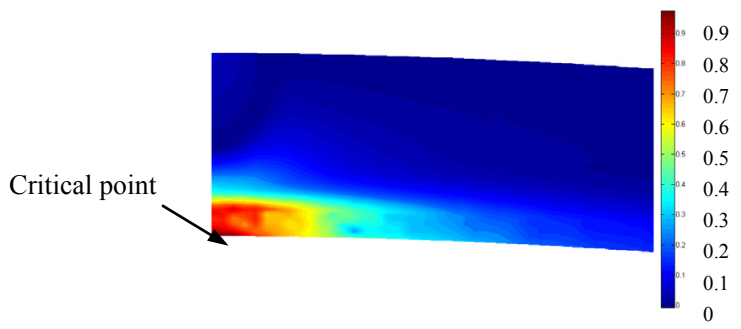

c)

Figure 3: Contour plots of the safety factor $(\lambda)$ to determine the failure point: a) failure at the calcite layer due to contact stresses; b) failure initiated at the interface of two layers in the nacreous side; c) failure at the inner surface of the shell due to flexural stress.

\subsubsection{Failure due to contact stresses}

In this case, Hertzian contact stresses control the failure. The configuration near the contact point is similar to a sphere in contact with a half space. According to the contact stress field, the maximum principle stress occurs on the surface of the shell, right at the edge of the contact area, and is believed to be responsible for the formation of Hertzian conical crack [5]. Typically, this type of failure was predicted for shells with a thick calcite layer (fig. 3a).

\subsubsection{Failure at the interface of two layers in the nacreous side}

For thick shells with a thinner calcite layer, the model predicted failure at the calcite/nacre interface. In this case, the calcite/nacre interface was close enough to the surface to "feel" the contact stresses. Since nacre has lower strength than calcite, it is prone to failure although the stresses on the points of two sides of the interface do not differ remarkably. Therefore, failure is observed at the interface of two layers on the nacreous side (fig. 3b).

\subsubsection{Failure due to flexural stresses}

Flexural stresses are generated by bending of the shell. Flexural failure was predicted at the bottom of the nacreous layer (fig. 3c). Flexural failure where observed when the thickness of the shell was decreased. In this case, flexural failure competes with contact failure, and which of these dominates is a function 
of the material properties (and of the nacre microstructure) and function of the overall geometry of the shell.

\section{Multiscale optimization}

An optimization study on the seashell structure and materials was accomplished to investigate how seashell geometry and material are optimized to resist penetration. The finite element model explained in the preceding section was used as the basis of the analysis within the structural optimization framework. The whole geometry of the shell can be expressed in terms of four macroscale parameters, namely, $R, t, \beta$ and $\alpha_{c}$ In addition, the radius of contact load is another variable that expresses the load distribution. Since the load carrying capacity of the shell depends on the load contact area, both the thickness and the radius of curvature were normalized with respect to the contact radius

$$
\bar{R}=\frac{R}{r_{p}}, \bar{t}=\frac{t}{r_{p}}
$$

Consequently, the results of modeling and optimization can be uniformly scaled for any structure with similar geometry and loading conditions. In addition to the structural variables at macroscale, three normalized microscale variables, i.e., $\bar{t}_{t}, \bar{t}_{i}$ and $\bar{l}_{o}$ control the mechanical behavior of nacre. It was assumed that junction has the same thickness as the interface. The optimization problem can now be described as:

$$
\begin{array}{ll}
\operatorname{maximize} \quad \frac{P_{\text {Load }}}{r_{p}{ }^{2}}=f\left(\bar{R}, \bar{t}, \alpha_{c}, \bar{t}_{t}, \bar{t}_{i}, \bar{l}_{o}\right) \\
\text { subject to } \quad\left\{\begin{array}{l}
10<\bar{R}<800, \\
1<\bar{t}<40, \\
0.5<\alpha_{c}<0.95 \\
0.02<\bar{t}_{t}<2, \\
0.001<\bar{t}_{i}<0.5, \\
0.1<\bar{l}_{o}<0.5
\end{array}\right.
\end{array}
$$

Nelder-Mead simplex search method (Nelder and Mead [14]) was applied to optimize the shell with respect to all six parameters. Since NM algorithm is susceptible in being entrapped in local optima, it was started randomly at 100 initial simplexes within the design space to increase the chance of converging to the global optimum. As expected, the algorithm converged to various points within the design space because of complicated shape of the objective function. Six best results, which exhibit the highest strength of the shell, were selected and shown in table 1 . As seen in table 1, although the results do not converge to a 
certain point, all parameters fall into a small range compared to the boundaries assigned to each variable as per eqn (10). According to the results, the optimum shell might be selected from a narrow range of geometric and material variables rather than from a unique selection, as it is found in nature where seashells of the same type exist in various sizes. By looking at the results of the microscale variables, it is observed that the optimum tablet and interface dimensions reached the lower boundaries. It occurred because thinner and thinner tablet and interface increase the modulus and strength of nacre, thereby improving the nacreous material properties. On the other hand, these dimensions cannot decrease further because of fabrication constraints and also failure of the tablet, which happens due to high stress transfer within the tablets. This phenomenon is also observed in nature where tablet aspect ratio increases up to the point where it is the tablet to fracture rather than the organic interface. The variation in the size of the overlap is also in good agreement with existing information about columnar and sheet nacre. In addition, all achieved optimum results took place at the transition of two failure modes, i.e., Hertzian contact failure in calcite layer and failure in nacreous layer initiated at the interface of two layers. As a result, the best performance of the shell can be achieved when two modes of failure in two different layers coincide meaning that the shell makes the most of its materials to resist against penetration.

Table 1: $\quad$ Results of multiscale optimization including six variables.

\begin{tabular}{|c|c|c|c|c|c|c|c|}
\hline & \multicolumn{3}{|c|}{ Shell Geometry } & \multicolumn{3}{|c|}{ Microstructure } & \multirow{2}{*}{$\begin{array}{c}\mathrm{P}_{\max } \\
/\left(\mathrm{r}_{\mathrm{p}}\right)^{2}(\mathrm{GPa})\end{array}$} \\
\hline & $\mathrm{R}^{*}$ & $\mathrm{t}^{*}$ & $\alpha_{c}$ & $\mathrm{t}_{\mathrm{t}}{ }^{*}$ & $\mathrm{t}_{\mathrm{i}}{ }^{*}$ & $1_{0}^{*}$ & \\
\hline Run \#1 & 366 & 8.5 & 0.63 & 0.02 & 0.001 & 0.44 & 7.354 \\
\hline Run \#2 & 333 & 8.5 & 0.62 & 0.02 & 0.001 & 0.34 & 7.298 \\
\hline Run \#3 & 428 & 9.4 & 0.66 & 0.02 & 0.003 & 0.2 & 7.207 \\
\hline Run \#4 & 407 & 11 & 0.78 & 0.02 & 0.003 & 0.36 & 6.143 \\
\hline Run \#5 & 412 & 15.8 & 0.34 & 0.05 & 0.012 & 0.4 & 6.097 \\
\hline Run \#6 & 391 & 17.2 & 0.37 & 0.07 & 0.038 & 0.44 & 5.934 \\
\hline
\end{tabular}

\section{Penetration tests on actual seashells}

In order to obtain an actual estimate of the force required to penetrate an actual shell, and to verify the failure mechanisms discussed above, experiments were performed on actual shells of red abalone to investigate their response to penetration. Two intact shells of red abalone were purchased from a shell shop (Specimen shells, Halifax NS) and kept in water to maintain hydration. For the experiments, the shells were laid on a custom made fixture (fig. 4) in a mechanical loading machine (MTS Systems Corporation, Eden Prairie, MN). The shells were punctured at their apex via an indenter connected to the load cell of the mechanical loading machine. A tungsten carbide ball of $1 \mathrm{~mm}$ in diameter was fixed on the tip of the indenter to transfer the load to the shell without deformation of the contact area. The inner surface of the shell underneath the 


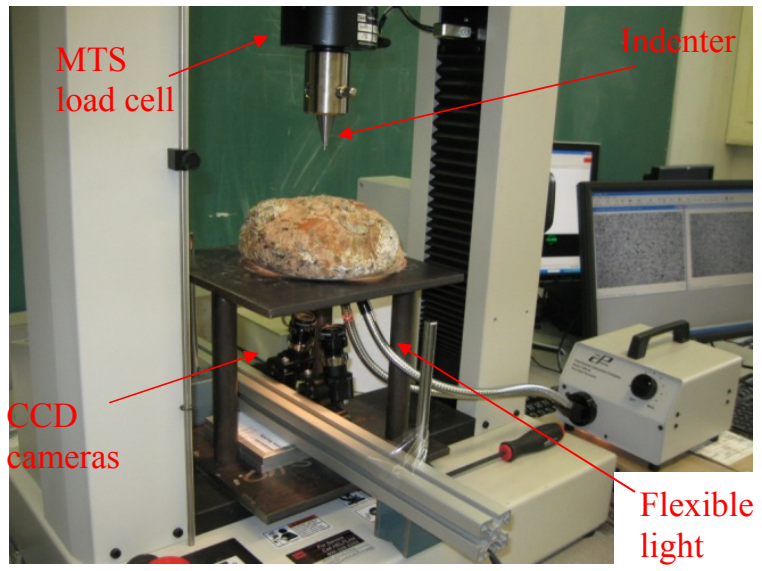

Figure 4: Experiment configuration.

indentation site was imaged through a hole in the base plate with two CCD cameras. Flexible links carrying lights at their tips were employed to illuminate the area inside the shell (fig. 4). During the experiments, the crosshead of the loading stage was moved down with a rate of $0.5 \mathrm{~mm} / \mathrm{sec}$.

Load versus crosshead displacement curves of the two experiments are shown together in fig. 5; the results of the first experiment is chosen to explain the failure behavior of the shell. At the beginning, the indenter penetrated into the calcite material. Calcite in the red abalone shell appeared to contain voids possibly generated by parasites. When the indenter was pressed against the calcite layer, it crushed the porous material underneath the contact region. At point $\mathrm{A}$, the shoulder of the indenter, which comprised the tungsten carbide ball, touched the surface of the calcite layer; at this moment, both the ball and the tip of the indenter were carrying the load. After this point, the load had to be increased to penetrate into the calcite material. However, with the same amount of load the shell started to bend and the flexural stresses at the inner surface of the shell rose until point $\mathrm{B}$, where a tiny crack was detected in nacre. The test was continued after point $C$ while acquiring photos from the inner surface of the shell to investigate the failure behavior. The flexural crack at the inner surface of the shell opened until the point where the indenter pierced more deeply into the calcite material. At this instant, the influence of Hertzian contact stresses reached the interface of two layers and nacreous material felt the high Hertzian contact stresses. As a result, the conical crack propagated into the nacreous layer and moved toward the inner surface of the shell where it finally merged with the flexural crack and then, formed a circular crack on the surface. As a result, a conical piece of the structure was cut out of the shell and loading was stopped. In both experiments, the three modes of failure were observed. The calcite failed partially at the beginning of the test. Then, the flexural crack was detected in the inner surface of the shell. After this point, the conical crack initiated inside the structure propagating in the nacreous layer toward the inner surface of the shell 


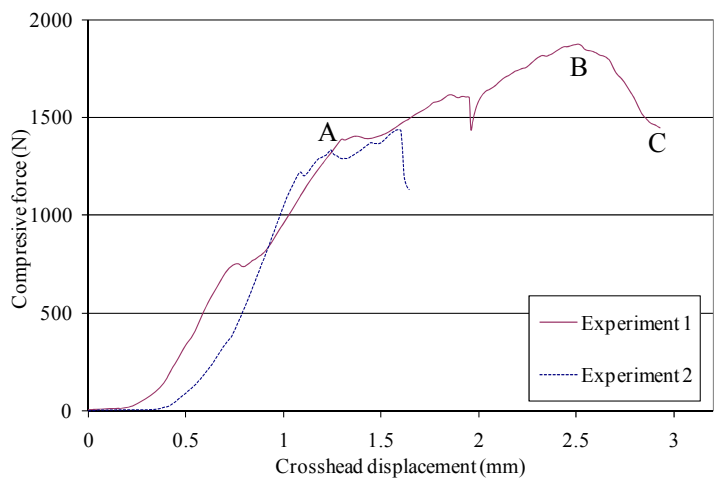

Figure 5: Load-crosshead displacement curve of the three experiments.

Table 2: $\quad$ Comparison of maximum load of modeling and experiments.

\begin{tabular}{|l|c|c|c|c|c|}
\hline & \multirow{2}{*}{$\mathrm{R}(\mathrm{cm})$} & \multirow{2}{*}{$\mathrm{t}(\mathrm{mm})$} & \multirow{2}{*}{$\alpha_{\mathrm{c}}$} & \multicolumn{2}{|c|}{ Load (N) } \\
\cline { 5 - 6 } & & & & Modeling & Experiment \\
\hline Experiment \# 1 & 12 & 8 & 0.45 & 1729 & 1863 \\
\hline Experiment \# 2 & 11 & 4 & 0.4 & 1097 & 1457 \\
\hline
\end{tabular}

where it merged with the flexural crack. Concurrent propagation of flexural and Hertzian conical cracks validates the optimization results elucidating that the best performance of the shell is achieved when two modes of failure coincide. Using the geometric information of the two samples of red abalone seashell, a finite element model, as described explained in the preceding sections, was used to estimate the maximum load that the shells can resist before failure. Table 2 compares FEM results with those of experiments. Considering all assumptions made in the modeling, the agreement between the experiment and the model is remarkably good.

\section{Conclusions}

Multiscale modeling and optimization of seashell structure and material were examined in this work. The geometry of the shell was modeled at the macroscale, and the micromechanics of the nacreous layer were also included. As a result of modeling, different failure modes could be obtained depending on the geometry of the shell. Optimization studies on the seashell model revealed that whenever two failure modes in different layers coincide, the shell shows its best performance in resisting against sharp penetration. In this case, the shell structure exploits the material capabilities at their full extent and distributes the stress into two different regions in order to avoid stress concentration. In addition, all parameters converged to a narrow range within the design space rather than converging to a single point. From the experiments performed on the two shells of red abalone, it can be concluded that the actual seashell arranges its microstructure design to fully exploit its materials and postpone failure, a result 
that was also obtained from the optimization study. The crack propagated through the thickness of the shell in three different failure modes. In addition, composed of typical ceramic material, the seashell could support up to $1900 \mathrm{~N}$ when loaded via a sharp indenter, which is surprisingly high compared to its size and structure.

\section{References}

[1] Mayer, G., Rigid biological systems as models for synthetic composites. Science, 310(5751), pp. 1144-1147,2005.

[2] Barthelat, F., et al., On the mechanics of mother-of-pearl: A key feature in the material hierarchical structure.Journal of the Mechanics and Physics of Solids, 55(2), pp. 306-337, 2007.

[3] Meyers, M.A., et al., Mechanical strength of abalone nacre: Role of the soft organic layer.Journal of the Mechanical Behavior of Biomedical Materials, 1(1), pp. 76-85, 2008.

[4] Sarikaya, M. \& I.A. Aksay, Biomimetics : design and processing of materials (aip series in polymers and complex materials), American Institute of Physics: New York,1995.

[5] Fischer-Cripps, A.C., Introduction to contact mechanics, Springer: New York, 2007.

[6] Caspi, E.N., et al., On the structure of aragonite. Acta Crystallographica Section B: Structural Science, 61(2), pp. 129-132, 2005.

[7] Currey, J.D., Mechanical properties of mother of pearl in tension. Proceedings of the Royal Society of London Series B-Biological Sciences, 196(1125), pp. 443-463, 1977.

[8] Kotha, S.P., Y. Li, \& N. Guzelsu, Micromechanical model of nacre tested in tension.Journal of Materials Science, 36(8), pp. 2001-2007, 2001.

[9] Wang, R.Z., et al., Deformation mechanisms in nacre. Journal of Materials Research, 16(9), pp. 2485-2493, 2001.

[10] Jager, I. \& P. Fratzl, Mineralized collagen fibrils: A mechanical model with a staggered arrangement of mineral particles. Biophysical Journal, 79(4), pp. 1737-1746, 2000.

[11] Barthelat, F., et al., Mechanical properties of nacre constituents and their impact on mechanical performance.Journal of Materials Research, 21(8), pp. 1977-1986, 2006.

[12] Barthelat, F. \& H.D. Espinosa, An experimental investigation of deformation and fracture of nacre-mother of pearl.Experimental Mechanics, 47(3), pp. 311-324, 2007.

[13] Lawn, B., et al., Failure modes in ceramic-based layer structures: A basis for materials design of dental crowns.Journal of the American Ceramic Society, 90(6), pp. 1671-1683, 2007.

[14] Nelder, J.A. \& R. Mead, A simplex-method for function minimization. Computer Journal,7(4), pp. 308-313, 1965. 